\title{
Método de Monte Carlo aplicado a economicidade do cultivo de tilápia-do-Nilo em tanques-rede
}

\author{
Simões, D. ${ }^{1}$ e Gouvea, A.C.F. ${ }^{1}$
}

'Centro Estadual de Educação Tecnológica Paula Souza. Botucatu. Brasil.

\section{PalaVRas ChaVE ADICIONAIS}

\section{Aquicultura.}

Custos.

Incerteza.

Oreochromis.

Viabilidade econômica.

\section{ADDITIONAL KEYWORDS}

Aquaculture.

Cost.

Uncertainty.

Oreochromis.

Economic viability.

\section{INFORMACIÓN}

Cronología del artículo.

Recibido/Received: 30.4 .2014

Aceptado/Accepted: 24.11.2014

On-line: 16.3 .2015

Correspondencia a los autores/Contact e-mail:

simoesdanilo@yahoo.com.br

\section{INTRODUÇÃO}

O cultivo de peixes em tanques-rede faz parte de uma categoria chamada de cultivo intensivo, tendo apresentado alto grau de desenvolvimento em várias partes do mundo e poderá ser uma importante opção disponível aos piscicultores brasileiros para a criação de espécies economicamente viáveis (Paiva et al., 2008).

A criação de peixes em sistema intensivo de produção, é uma modalidade, com alta e contínua renovação de água visando manter a qualidade da água dentro dos tanques-rede e, remover os metabólitos e dejetos produzidos pelos peixes. Trata-se de excelente alternativa para o aproveitamento de corpos d'água inexplorados pela piscicultura convencional (Colt, 1991).

Para Silva et al. (2012) os novos modelos de produção e o crescimento do consumo de peixes pro- movem o avanço da aquicultura, que por sua vez, demandam informações para tomada de decisão sobre a viabilidade econômica e a rentabilidade dos empreendimentos aquícolas. O fator primordial de que depende o desenvolvimento da piscicultura é o seu sucesso econômico (Lima et al., 2009). Para tanto, se faz necessário o conhecimento e análise dos custos de operação dos projetos de cultivo de peixes (Saint-Paul, 1986).

Os custos e benefícios associados ao fluxo de caixa de projetos de investimento normalmente são conhecidos, caracterizando o que se conhece como procedimento de análise determinística; apesar de sua praticidade, leva a uma simplificação ou superestimativa de informações que nem sempre são conhecidas com certeza no momento da análise, como preços, quantidades e rendimentos, entre outros (Cordeiro et al., 2010).

Para evitar essas incertezas, Mendonça et al. (2009) relatam que uma das formas mais utilizadas na opera- 
cionalização da análise de risco é o método de simulação de Monte Carlo, que consiste na utilização de distribuições de probabilidades das variáveis consideradas incertas (variáveis de entrada), complementado por Corrar (1993) que também recomenda que em condiçõos de incerteza, o emprego do modelo de simulação de Monte Carlo.

No método de Monte Carlo, as principais fontes de incerteza do projeto, tais como: receitas, taxas de desconto, custos e despesas, impostos e depreciação, entre outras, podem servir como variáveis de entrada para a simulação (Oliveira e Pamplona, 2012).

A simulação de Monte Carlo é uma importante ferramentas na avaliação de risco, e que possui duas propriedades; a consideração simultânea de ameaças e oportunidades, e a probabilidade de selecionar vários critérios para análise (Rezaie et al., 2007).

A simulação de Monte Carlo se baseia na geração de uma série de número pseudoaleatórios previamente estabelecidos com seus valores distribuídos de acordo com uma função e parâmetros identificados na amostra a ser analisada, para avaliar seu comprimento em relação à distribuição dos elementos da amostra (Martins et al., 2012).

Diante do exposto, objetivou-se avaliar a economicidade do cultivo de tilápia-do-Nilo Oreochromis spp. em condições de risco com o uso da simulação pelo método de Monte Carlo.

\section{MATERIAL E MÉTODOS}

Para a estimativa da economicidade do cultivo de tilápias, foram considerados dados referentes à implantação do projeto de investimento num represamento em uma propriedade particular, localizado nas coordenadas geográficas $22^{\circ} 22^{\prime}$ de Latitude Sul e $48^{\circ} 45^{\prime}$ de Longitude Oeste, no município de Botucatu, Estado de São Paulo - Brasil, com altitude média de 483 metros acima do nível do mar. De acordo com a classificação climática de Köppen, o clima é Cwa, caracterizado como temperado úmido, com inverno seco e verão quente (Centro de Pesquisas Meteorológicas e Climáticas Aplicadas à Agricultura, 2013).

Foram considerados para o estudo a instalação de 10 tanques-rede de dimensões 3,0 $\mathrm{m} \times 3,0 \mathrm{~m} \times 1,5 \mathrm{~m}$ e com volume útil de $12 \mathrm{~m}^{3}$, construídos em aço galvanizado revestido de policloreto de vinil (PVC) de alta aderência, com malha de $6 \mathrm{~mm}$ entre nós, com estrutura para ancoramento, flutuadores de PVC de 30 litros com proteção anti-UV na cor amarelo, os quais recebem aeração forçada originária de um soprador de $2 \mathrm{cv}$. Internamente foram instalados comedouros, fixados em todo o perímetro interno dos tanques-rede.

A instalação dos tanques-rede foi com alinhamento perpendicularmente à margem do represamento com profundidade média de $2 \mathrm{~m}$, presos a cabos e divididos em dois alinhamentos de tanques-rede, com espaçamento de $1 \mathrm{~m}$ entre eles. A espécie cultivada é a tilápiado-Nilo (Oreochromis spp.), devido à possibilidade do cultivo durante o ano todo e também por apresentar potencial para a piscicultura em nível mundial. O sis- tema produtivo utilizou somente tanques-rede e compreendeu três fases de desenvolvimento:

Fase 1- aquisição dos alevinos de uma piscicultura comercial, com comprimento médio de $0,65 \mathrm{~cm}$ e massa média de 0,25 g estocados na densidade de 340 peixes por metro cúbico. Durante um período de 40 dias, foi utilizada ração comercial para a alimentação, com 42,0 \% de proteína bruta e $500 \mathrm{mg} \mathrm{kg}^{-1}$ de vitamina C, numa proporção de $5 \%$ da massa viva, fracionada em quatro vezes ao dia. Nessa fase, a taxa de mortalidade de aproximadamente 10,0\%;

Fase 2- refere-se à terminação, com a alimentação realizada três vezes ao dia. A densidade de estocagem foi de 130 peixes por metro cúbico, onde receberam uma ração formulada com 32,0 \% de proteína bruta e $300 \mathrm{mg} \mathrm{kg}^{-1}$ de vitamina C, na proporção de 3,0 \% da massa viva, até atingirem entre 60 e 100 g, em aproximadamente 60 dias;

Fase 3- após os peixes atingirem um tamanho e massa uniforme, ocorreu o povoamento dos tanquesrede para o decrescimento/terminação, onde a alimentação foi à base de ração comercial com 28,0 \% de proteína bruta e $200 \mathrm{mg} \mathrm{kg}^{-1}$ de vitamina C, destinada à peixes que se encontram nessa fase de produção, especialmente para tilápias, cultivadas em sistema extensivo, até o abate.

A despesca era realizada ao final do ciclo produtivo, caracterizado como uma variável de entrada do modelo probabilístico, ou seja, passível de simulação, considerando-se ainda um intervalo de dias entre ciclos (também variável de entrada), o qual se faz necessário após a despesca para a realização de manutenções preditivas, preventivas ou até mesmo corretivas. Igualmente, determinou-se como variável de entrada, a capacidade de produção $(\mathrm{kg})$ de tilápia-do-Nilo a cada ciclo. A biometria, o povoamento, a classificação e a necessidade a redistribuição dos peixes, foram realizadas quatro vezes durante cada ciclo produtivo.

A análise econômica foi constituída a partir da obtenção do custo total de produção (CTP), por meio da metodologia de custo utilizada pelo Instituto de Economia Agrícola (IEA), proposta por Matsunaga et al. (1976), classificado em:

Custo operacional efetivo (COE), onde ponderouse os custos com: alevinos; análises d'agua; assistência técnica; corretivos e fertilizantes; energia elétrica; materiais para manutenção; outorga e licenciamento ambiental; ração; registro de aquicultor; reparos e manutenção; salário de um colaborador; taxas de regularização do projeto; e telecomunicações;

Custo operacional total (COT), que inclui ao somatório do COE a depreciação; os encargos sociais (contribuição ao INSS, férias, FGTS e outras despesas) sendo utilizado para essa estimativa o percentual de 43,0\% do gasto com mão de obra permanente; impostos; custo total de produção (CTP), resultante do somatório do COT e da remuneração do capital investido.

Esses custos foram expressos em dólar comercial americano, por ser utilizado como moeda internacional de referência, segundo Simões et al. (2012) e utilizada 
como parâmetro para o mercado financeiro (Coelho Junior et al., 2008). Foi considerado como taxa de câmbio o preço da moeda estrangeira oficial do Banco Central do Brasil (PTAX 800) a preço de venda, medido em unidades e frações da moeda nacional, que era de $\mathrm{R} \$$ 1,9861 em 09/04/2013 (Banco Central do Brasil, 2013).

Os indicadores de rentabilidade foram os utilizados por Sanches et al. (2013), sendo esses: receita bruta (RB); lucro operacional (LO); e margem bruta (MB). O ponto de equilíbrio contábil (PEC) foi obtido pela divisão dos custos fixos totais, pela margem de contribuição, expresso em quilogramas produzido de tilápia-do-Nilo e em unidades monetárias (US\$).

A incorporação de risco ao projeto de investimento financeiro deu-se a partir do método estocástico de Monte Carlo, com simulações realizadas por meio do software @Risk para Excel 6.2 (Palisade Corporation, 2013), com distribuição de probabilidade estratificada. O gerador de números randômicos utilizado foi o Mersenne Twister conforme Matsumoto e Nishimura (1998). A quantidade de números pseudoaleatório gerados foi determinado em função da ótima convergência dos valores simulados.

Em decorrência do não conhecimento das distribuições de cada variável, que possui a maior implicação sobre o resultado financeiro do projeto foi aplicada a distribuição triangular. Lyra et al. (2010) discorrem que diante da dificuldade envolvida na identificação das distribuições de probabilidade de cada uma das variáveis mais relevantes, esse é o procedimento usual. A distribuição triangular foi definida pelo nível médio mais provável ou moda, por um nível mínimo e um nível máximo, o que é importante quando não se dispõe de conhecimento suficiente sobre as variáveis, de acordo com Ponciano et al. (2004).

Devido à incerteza da trajetória que a taxa de juros praticada pelo mercado financeiro pode apresentar, foi adotado o modelo autorregressivo integrado com médias móveis (ARIMA) proposto por Box e Jenkins (1970) pelo critério de seleção Bayes Information Criterion (BIC) desenvolvido por Schwarz (1978). Dessa forma, utilizou-se dados da série temporal econômicofinanceira referente ao sistema especial de liquidação e de custódia (SELIC) disponibilizada pelo Banco Central do Brasil (2014), observada entre janeiro de 2008 e janeiro de 2014, para realizar a previsão futura da taxa de desconto a ser utilizada no estudo. O mesmo modelo, fonte de dados e período foi adotado para projetar a inflação, com base no índice nacional de preços ao consumidor amplo (IPCA), com o propósito de atualizar o valor da depreciação e dos impostos pagos durante a série do fluxo de caixa.

Neste modelo probabilístico foram consideradas 8 variáveis de entrada (inputs) que relacionadas entre si, influenciam diretamente na formação da variável dependente Receita Bruta (RB), sendo essas: quantidade de dias do ciclo de cultivo; intervalo de dias entre ciclos; produção por ciclo $(\mathrm{kg})$; investimento financeiro; custo total de produção; valor de venda esperado; SELIC; e IPCA. Para definir o valor mínimo, modal e máximo dos inputs, delimitou-se uma variante de $-20 \%$ a $+20 \%$ dos valores determinísticos, exceto para as taxas SELIC e IPCA que foram projetadas através das médias moveis.

O fluxo de caixa compreendeu um período de 7 anos, sendo esse determinado em função da vida útil dos tanques-rede ser 16 ciclos de cultivo. Os indicadores de viabilidade econômica, considerados como variáveis de saída (outputs) foram: valor presente líquido (VPL) e taxa interna de retorno (TIR), ambos conforme o proposto por Murta et al. (2013); Payback descontado de acordo com Siqueira et al. (2011); e a relação benefício/custo (R B/C), consoante Maneschy (2009), os quais são comumente utilizados para análises de investimentos financeiros.

\section{RESULTADOS E DISCUSSÕES}

Os investimentos financeiros para a implantação de 10 tanques-rede para o cultivo de tilápia-do-Nilo, na região Centro-Oeste do Estado de São Paulo - Brasil, são apresentados na tabela I. O valor modal simulado para o investimento inicial referente à implantação do projeto foi US\$24188,15. Desse montante 79,6 \% foram destinados à aquisição de equipamentos necessários para a implantação do sistema de piscicultura, sendo que 56,8 \% referem-se à aquisição dos tanques. Ayroza et al. (2011), Furlaneto et al. (2010) e Campos et al. (2007) obtiveram em seus estudos percentuais acima de $60 \%$ somente com a aquisição dos tanquesrede, fato que pode ser um restritivo para pequenos ou médios aquicultores, sobretudo àqueles que almejam realizar o investimento com recursos próprios. Quanto aos dispêndios com a regularização do projeto, foram necessários 16,2\% do valor total do capital.

Conforme o dimensionamento proposto, o custo operacional efetivo (COE) apresentando na tabela II, representou $81,7 \%$ do custo total de produção (CTP), tendo como valor mais prováve 11,33 US\$ $\mathrm{kg}^{-1}$ de tilápia-do-Nilo produzida em tanques-rede, valor próximo ao estimado por Lima et al. (2009) em estudo realizado em 8 empreendimentos localizados no Estado da Paraíba. Dentre os itens que compõem o custo total de produção, a ração adquirida a um preço médio de US\$ 0,53, foi o insumo com maior representatividade $(42,4 \%)$ corroborando Oliveira et al. (2010) os quais afirmam que o elevado custo com ração na piscicultura é fator que merece atenção por parte de pesquisadores e produtores. Contudo Furlaneto et al. (2006) salientam que o uso de ração de boa qualidade e alta digestibilidade, aliado a um programa alimentar eficiente contribui para o bom desempenho e saúde dos animais, além de reduzir a poluição do meio ambiente.

Ainda, entre os itens que apresentaram maior significância na estimativa do custo total de produção, têm-se as despesas com mão de obra (salário e encargos sociais) com 11,9 \% e a aquisição dos alevinos em $6,3 \%$ do custo total de produção. Por conseguinte, o custo de ração, aquisição de alevinos e despesas com mão de obra, representaram aproximadamente $60,6 \%$ do custo por ciclo para o cultivo de tilápia-do-Nilo em 10 tanques-rede.

A simulação pelo método de Monte Carlo possibilitou a geração de valores com probabilidade de 
Tabela I. Premissas de investimento financeiro para o cultivo de tilápia-do-Nilo (Assumptions of financial investment for the cultivation of the Nile tilapia).

\begin{tabular}{|c|c|c|c|c|}
\hline Descrição & $\begin{array}{l}\text { Valor total } \\
\quad \text { (US\$) }\end{array}$ & $\begin{array}{l}\text { Vida útil } \\
\text { (anos) }\end{array}$ & $\begin{array}{l}\text { Depreciação } \\
\text { (US\$ ano 1) }\end{array}$ & $\begin{array}{c}\text { Remuneração do capital } \\
\text { (US\$ ano 1) }\end{array}$ \\
\hline \multicolumn{5}{|l|}{ Serviços } \\
\hline Análises da água & 372,79 & - & - & - \\
\hline Outorga e licenciamento ambiental & 1562,44 & - & - & - \\
\hline Registro de aquicultor & 161,11 & - & - & - \\
\hline Taxas de regularização & 1813,99 & - & - & - \\
\hline \multicolumn{5}{|l|}{ Instalações } \\
\hline $\begin{array}{l}\text { Biometria, triagem, contagem, } \\
\text { despesca e povoamento }\end{array}$ & 126,13 & 5 & 25,23 & 13,24 \\
\hline Tanques-rede & 13750 & 7 & 2750 & 1443,75 \\
\hline Sistema para ancoragem & 747 & 5 & 149,40 & 78,44 \\
\hline Panagem para alevinagem & 302,10 & 5 & 60,42 & 31,72 \\
\hline \multicolumn{5}{|l|}{ Equipamentos para manejo } \\
\hline Balança & 376,96 & 5 & 75,39 & 39,58 \\
\hline Caixa d'agua & 223,50 & 5 & 44,70 & 23,47 \\
\hline Mesa para seleção & 66,05 & 5 & 13,21 & 6,94 \\
\hline \multicolumn{5}{|l|}{ Equipamentos para aeração } \\
\hline Soprador & 957,35 & 5 & 191,47 & 100,52 \\
\hline Difusor $20 \mathrm{~cm}$ & 60,42 & 5 & 12,08 & 6,34 \\
\hline Material hidráulico & 356,98 & 5 & 71,40 & 37,48 \\
\hline Material elétrico & 151,12 & 5 & 30,22 & 15,87 \\
\hline \multicolumn{5}{|l|}{ Equipamentos de controle } \\
\hline Kit de análise & 302,10 & 7 & 45,31 & 31,72 \\
\hline Termômetro & 60,42 & 7 & 9,06 & 6,34 \\
\hline Disco de secchi & 20,14 & 7 & 3,02 & 2,11 \\
\hline Balança para biometria & 60,42 & 5 & 12,08 & 6,34 \\
\hline Balança para ração & 100,70 & 5 & 20,14 & 10,57 \\
\hline Puçá & 75,52 & 5 & 15,10 & 7,93 \\
\hline Baldes & 61 & 5 & 12,2 & 6,41 \\
\hline Trena & 10,07 & 5 & 2,01 & 1,06 \\
\hline Barco de alumínio & 1402,80 & 5 & 280,56 & 147,29 \\
\hline Kit de ferramentas & 40,34 & 5 & 8,07 & 4,24 \\
\hline \multicolumn{5}{|l|}{ Equipamentos de escritório } \\
\hline Mesas & 84,80 & 20 & 4,24 & 8,90 \\
\hline Cadeiras & 68,50 & 20 & 3,43 & 7,19 \\
\hline Aparelho telefônico & 42,70 & 10 & 4,27 & 4,48 \\
\hline Microcomputador & 705,10 & 5 & 141,02 & 74,04 \\
\hline Impressora & 125,60 & 5 & 25,12 & 13,19 \\
\hline
\end{tabular}

ocorrência similar à situações reais, por meio de números pseudoaleatórios, eliminando a possibilidade tendenciosa, sobretudo devido à ótima convergência do modelo probabilístico com 33900 situações, garantindo uma maior segurança para o número de iterações realizadas.

Ao considerar a massa média de abate de 550 g, sendo essa obtida pelo aquicultor e comumente adquirida pelo mercado consumidor, a capacidade probabilística de cultivo foi de $14500 \mathrm{~kg}$ de tilápia-do-Nilo produzidas a cada ciclo. Durante a vida útil do projeto será possível a realização de 18 ciclos produtivos. O período mais provável de cultivo foi de 145 dias, considerando 10 dias como modal para o intervalo entre ciclos, corroborando os estudos realizados por Marengoni (2006) e Conroy (2000) os quais produziram tilápias com
135 dias. Contudo esse período é suscetível, devido à massa de abate desejável, alimentação, procedência e sanidade dos alevinos, forma de manejo, entre outros.

A receita bruta $(\mathrm{RB})$ propiciada com a venda de tilápia-do-Nilo para a indústria de filetagem, foi de US\$ 24650, com um valor de venda esperado de 1,70 US\$ $\mathrm{kg}^{-1}$, sendo esse o mesmo esperado por Furlaneto et al. (2010), a qual resultará num lucro operacional (LO) de US\$ 5317,96 a cada ciclo de cultivo. A margem bruta (MB) foi de 27,5\%, sendo essa superior ao relatado por Scorvo Filho et al. (2008) que estimaram uma margem média de 23,47 \% para 6 tipos de manejos para a criação de tilápia em tanques-rede. De acordo com Brabo (2013) a margem bruta é um indicador que deve ser entendido como a taxa de retorno para remunerar outros custos, além do desembolso e da depreciação. 
Tabela II. Custo operacional efetivo e total de produção para o cultivo de tilápia-do-Nilo (Effective operational cost and total production for the cultivation of the Nile tilapia).

\begin{tabular}{lcc}
\hline Custo operacional efetivo - COE & US\$ $\left(\right.$ ciclo $\left.^{-1}\right)$ & (\%) CTP \\
\hline Salário do colaborador & 2100 & 10,86 \\
Alevinos Oreochromis & 1211,70 & 6,27 \\
Ração & 8198,56 & 42,41 \\
Energia elétrica & 507,50 & 2,63 \\
Corretivos e fertilizantes & 655,21 & 3,39 \\
Assistência técnica & 823,55 & 4,26 \\
Registro de aquicultor & 62,65 & 0,32 \\
Reparos e manutenção & 604,17 & 3,13 \\
Materiais para manutenção & 94,25 & 0,49 \\
Telecomunicações & 87,00 & 0,45 \\
Outorga e licenciamento ambiental & 607,62 & 3,14 \\
Taxas de regularização & 705,44 & 3,65 \\
Análises da água & 144,97 & 0,75 \\
\hline Custo operacional total - COT & \\
\hline Depreciação & 1538,88 & 7,96 \\
Encargos sociais & 201,06 & 1,04 \\
Impostos & 665,55 & 3,44 \\
\hline Subtotal & \\
\hline COE + COT & 18208,11 & 94,19 \\
Remuneração do capital & 1123,94 & 5,81 \\
Custo total de produção - CTP & 19332,04 & 100,00 \\
\hline
\end{tabular}

O ponto de equilíbrio contábil pode ser expresso em unidades produzidas ou unidades financeiras, com a finalidade de quantificar a venda mínima que o empreendimento deverá realizar para não ocorrer perda financeira. Portanto, são contemplados todos os custos e dispêndios indispensáveis à obtenção de receita. Assim, foi necessário um cultivo aproximado de 9400 quilogramas de tilápia-do-Nilo, o que representa em torno de US\$ 16000 de venda mínima necessária, para que se possa cobrir todas as despesas com o projeto de investimento, valores esses estimados com uma margem de segurança de aproximadamente $35,1 \%$. De acordo com Raimundini (2008) o ponto de equilíbrio constitui uma das ferramentas de análise de custos mais utilizadas em qualquer atividade econômica, uma vez que hoje são exigidas tomadas de decisões complexas para manter e aumentar a lucratividade e o desempenho de um empreendimento.

Os riscos financeiros de um projeto estão relacionados com as incertezas do mercado, sendo esses minimizados por projeções de valores, obtidos através de modelos estatísticos. Assim, com base no modelo ARIMA pelo critério BIC, foi possível modelar uma série temporal assumindo uma previsão de $10,5 \%$ para a taxa de desconto e 5,4\% para o IPCA, fato que exprimi a probabilidade do fluxo de caixa numa data futura.

Bentes-Gama et al. (2005) relatam que de maneira geral, os benefícios e custos associados ao fluxo de caixa de projetos de investimento normalmente são considerados conhecidos, caracterizando o que se conhece como procedimento de análise determinística; que, apesar de sua praticidade, leva a uma simplificação e, ou, a superestimativa de informações que nem sempre são conhecidas com certeza no momento da análise, como preços, quantidades, e rendimentos, entre outros. Por meio do método de Monte Carlo, na figura 1 é demonstrado a simulação dos recebimento e pagamentos realizados pelo aquicultor durante a vida útil do projeto de investimento, sendo dessa forma considerado como um fluxo de entradas e saídas, as quais são distintas em decorrência do número de ciclos de cultivo ao ano, que concomitantemente estão vinculados ao período do ciclo produtivo e o intervalo entre os ciclos.

A análise de sensibilidade evidenciou que dentre as 8 variáveis de entrada analisadas pela distribuição triangular, somente 5 exercem influência na viabilidade econômico-financeira do projeto de investimento. Desse forma, a quantidade cultivada por ciclo possui o maior efeito sobre o VPL, sendo que a redução deste em $20 \%$ decresceria o valor modal do VPL em 123,1 \%. Nesse sentido, a quantidade de tilápia-do-Nilo cultivada por ciclo mostra-se como fator decisivo para a economicidade do projeto de investimento aquícola, o que indica a necessidade de medidas mitigadoras para minimizar possíveis riscos, principalmente em relação ao manejo e a taxa de mortalidade. O VPL esperado para o cultivo de tilápia-do-Nilo foi de US\$ 42565,95. Na figura 2 são apresentados os coeficientes linear de Spearman das variáveis de entrada que mais impactaram no VPL. Este coeficiente é o mais antigo e também o mais conhecido para calcular o coeficiente de correlação entre variáveis mensuradas em nível ordinal (Lira e Chaves Neto et al., 2006). O grau de correlação $\left(R^{2}=0,989\right)$ indicou uma correlação perfeita, corroborando que a quantidade cultivada por ciclo $(\mathrm{kg})$ e o valor de venda esperado (US\$) são diretamente proporcionais em relação ao VPL. Todavia, como poderia presumir o CTP e os dias de ciclo de necessários para o cultivo provocam um efeito inverso no VPL para projeto de investimento do cultivo de tilápia-do-Nilo em tanques-rede.

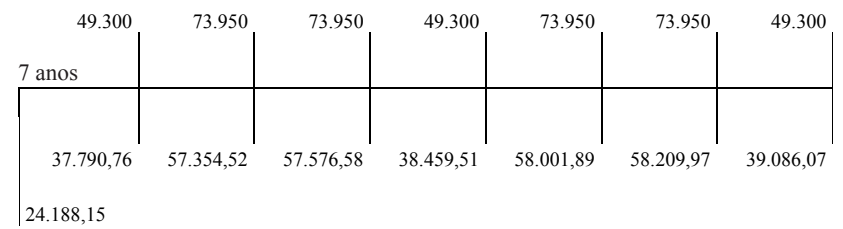

Figura 1. Diagrama do fluxo de caixa do projeto de investimento para o cultivo de tilápia-do-Nilo (Diagram of cash flow of the investment project for the cultivation of Nile tilapia).

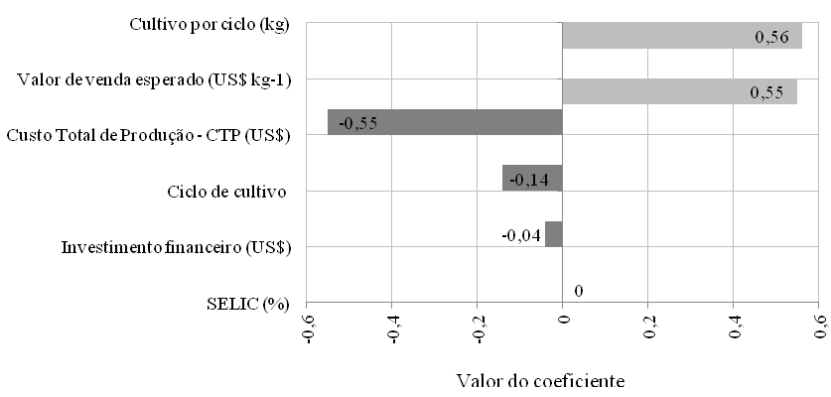

Figura 2. Coeficiente de correlação linear de Spearman das variáveis de entrada do modelo em relação ao VPL (Linear correlation coefficient of Spearman of the input of the model in relation to NPV). 


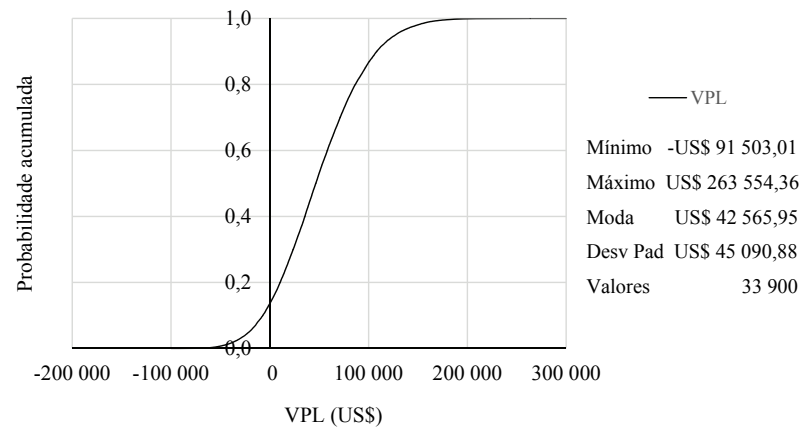

Figura 3. Frequência acumulada do VPL simulado para o cultivo de tilápia-do-Nilo (Cumulative frequency of NPV simulate of the cultivation Nile tilapia).

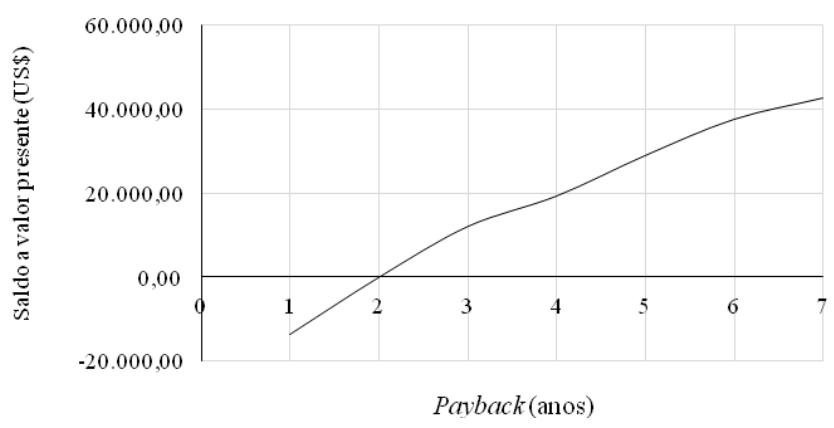

Figura 4. Período de recuperação do investimento para o cultivo de tilápia-do-Nilo (Recovery period of the investment of the cultivation Nile tilapia).

Na figura 3 é apresentada a distribuição de frequência triangular acumulada, obtida com a simulação pelo método de Monte Carlo, onde têm-se 81,4 \% de probabilidade do aquicultor obter um VPL entre US $\$ 0$ e 127 246. A probabilidade do VPL ficar abaixo de 0 deve ser observada com prudência, pois o percentual obtido sobre o histograma projetado é de $13,6 \%$. Castro et al. (2007) afirmam que com a análise determinística não seria possível inferir sobre essas possibilidades, pois ela não considera variáveis aleatórias e probabilísticas como na simulação por Monte Carlo.

Em relação à taxa interna de retorno (TIR) o percentual esperado é de $54,2 \%$, consideravelmente superior à SELIC. Portanto o cultivo de tilápia-do-Nilo em 10 tanques-rede, possibilita ao aquicultor uma remuneração do capital investido, superior aos títulos de renda fixa disponíveis no mercado financeiro. A análise de sensibilidade indicou que a produção por ciclo $(\mathrm{kg})$ e o valor de venda esperado (US\$) também são as variáveis que mais exercem impacto neste indicador de viabilidade econômica, corroboram a suscetibilidade dessas variáveis de entrada nos resultados financeiros do projeto em aquicultura para o cultivo de tilápia-doNilo, confirmada pela correlação $\mathrm{R}^{2}=0,975$.

Na figura 4 pode ser observado o payback descontado, ou seja, o tempo de recuperação do capital investindo considerando o fluxo de caixa descontado a uma taxa mínima de retorno esperada pelo aquicultor, sendo esse período estimado em 2,02 anos, próximo ao obtido por Furlaneto et al. (2010) que avaliou ser necessário seis ciclos com 150 dias para recuperação do investimento financeiro e inferior ao obtido por Brabo et al. (2013) que obteve um período de retorno de 6,5
Tabela III. Estatística descritiva dos indicadores de viabilidade econômica (Descriptive statistics of indicators of economic viability for the cultivation of the Nile tilapia).

\begin{tabular}{lcccc}
\hline Estatística & VPL & TIR & Payback & R B/C \\
\hline Mínimo & $-91503,01$ & $-65,9 \%$ & 0,00 & 0,68 \\
Máximo & 263554,36 & $254,8 \%$ & 7,00 & 2,13 \\
Média & 49017,69 & $60,7 \%$ & 1,87 & 1,19 \\
Desvio & 45090,88 & $38,6 \%$ & 1,34 & 0,18 \\
padrão & 0,29 & 0,243061 & 0,178682 & 0,398272 \\
Assimetria & 3,02 & 3,229784 & 2,827531 & 3,124053 \\
Curtose & 42565,95 & $54,2 \%$ & 0,00 & 1,14 \\
Moda & & & & \\
\hline Percentis & & & 0,00 & 0,92 \\
\hline $5 \%$ & $-20905,12$ & $-1,0 \%$ & 0,74 & 1,01 \\
$15 \%$ & 2495,85 & $21,4 \%$ & 1,15 & 1,07 \\
$25 \%$ & 17259,31 & $34,6 \%$ & 1,35 & 1,11 \\
$35 \%$ & 29687,37 & $45,3 \%$ & 1,54 & 1,16 \\
$45 \%$ & 40925,25 & $54,6 \%$ & 1,75 & 1,20 \\
$55 \%$ & 52096,93 & $64,2 \%$ & 1,25 \\
$65 \%$ & 64564,45 & $74,2 \%$ & 2,02 & 1,25 \\
$75 \%$ & 78325,62 & $85,4 \%$ & 2,41 & 1,31 \\
$85 \%$ & 96547,75 & $100,0 \%$ & 3,06 & 1,38 \\
$95 \%$ & 127245,77 & $126,2 \%$ & 4,61 & 1,50 \\
\hline
\end{tabular}

$\mathrm{VPL}=$ valor presente líquido; $\mathrm{TIR}=$ taxa interna de retorno; $\mathrm{R} / \mathrm{BC}=$ relação benefício/custo.

anos para um empreendimento com oito tanques-rede para o cultivo de tilápias.

A relação benefício/custo $(\mathrm{R} \mathrm{B/C})$ indicou que a expectativa de retorno para cada unidade de capital imobilizado no projeto foi de 1,16, ou seja, a cada um dólar investido o aquicultor terá US\$ 0,16 de retorno, corroborando a economicidade dos demais indicadores de viabilidade econômica.

Os projetos de investimento financeiro normalmente limitam-se à modelos determinísticos que podem resultar em incertezas. Mesmo com suas limitações, esses modelos são comumente aplicados pelos gestores ou tomadores de decisão, contudo, faz-se necessário a realização de uma análise das incertezas, ou seja, a concepção de um ambiente probabilístico que possibilite o monitoramento e o planejamento de respostas aos riscos dos projetos, gerando estimativas mais confiáveis.

Através da simulação dos indicadores de viabilidade econômica para o cultivo de tilápia-do-Nilo em tanques-rede, pode ser observado na tabela III os resultados estatísticos referentes às variáveis de saídas. A assimetria e curtose dos indicadores de viabilidade econômica permite considerar que as distribuições têm um padrão aproximadamente normal, respectivamente próximos de 0 e 3.

\section{CONCLUSÕES}

A análise de sensibilidade indicou uma correlação diretamente proporcional de 98,9\% para as variáveis quantidade cultivada e valor de venda esperado, em relação ao valor presente líquido do projeto de investimento financeiro para o cultivo de tilápia-do-Nilo. 
O investimento destinado à implantação de 10 tanques-rede para a produção de tilápia-do-Nỉo na região Centro-Oeste do Estado de São Paulo - Brasil é exequível, pois os indicadores de viabilidade comumente utilizados em projetos econômico-financeira, indicam que o empreendimento aquícola é economicamente viável.

A probabilidade da taxa interna de retorno ser abaixo da taxa de juros esperada para o modelo probabilístico é de 9,2\% e da relação benefício/custo ser nula é de $13,6 \%$.

Ao considerar 135 dias de cultivo e 10 de intervalo entre ciclos, serão necessários aproximadamente 5 ciclos para o aquicultor recuperar o capital investido para a produção de tilápia-do-Nilo em 10 tanques-rede.

\section{BIBLIOGRAFIA}

Ayroza, L.M. da Silva; Romagosa, E.; Ayroza, D.M. Rezende; Scorvo Filho, J.D. e Salles, F.A. 2011. Custos e rentabilidade da produção de juvenis de tilápia-do-Nilo em tanques-rede utilizando-se diferentes densidades de estocagem. Rev Bras Zootecn, 40: 231-239.

Bentes-Gama, M.M.; Silva, M.L.; Vilcahuamán, L.J.M. e Locatelli, M. 2005. Análise econômica de sistemas agroflorestais na Amazônia ocidental, Machadinho d'oeste-RO. Rev Árvore, 29: 401-411.

Banco Central do Brasil. 2013. Conversão de moedas. http://www4. bcb.gov.br/pec/conversao/conversao.asp (12/3/2013).

Banco Central do Brasil. 2014. Estatísticas econômico-financeiras. https://www3.bcb.gov.br/sgspub/pefi300/telaCtjSelecao.paint (12/3/2013).

Box, G. and Jenkins, G. 1970. Time series analysis: forecasting and control. $3^{\mathrm{a}}$ ed. Holden-Day. San Francisco. $592 \mathrm{pp}$

Brabo, M.F.; Flexa, C.E.; Veras, G.C.; Paiva, R.S.; Fujimoto, R.Y. 2013. Viabilidade econômica da piscicultura em tanques-rede no reservatório da usina hidrelétrica de Tucuruí, Estado do Pará. Informações Econômicas, 43: 56-64.

Colt, J. 1991. Aquacultural production systems. J Anim Sci, 69: 41834192.

Campos, C.M.; Ganeco, L.N.; Castellani, D. e Martins, M.I.E. 2007. Avaliação econômica da criação de tilápias em tanque-rede, município de Zacarias, SP. Informações Econômicas, 33: 265-271.

Castro, R. R.; da Silva, M.L.; Leite, H.G. e de Oliveira, M.L.R. 2007. Rentabilidade econômica e risco na produção de carvão vegetal. Cerne, 13: 353-359.

Centro de Pesquisas Meteorológicas e Climáticas Aplicadas a Agricultura. 2013. Clima dos municípios paulistas. http://www.cpa.unicamp.br (18/3/2013)

Coelho Júnior, L.M.; de Rezende, J.L.P.; de Oliveira, A.D.; Borges, L.A.C. e de Souza, A.N. 2008. Análise de investimento de um sistema agroflorestal sob situação de risco. Rev Cerne, 14: 368-378.

Conroy, G. 2000. Alteraciones asociadas con dos alimentos comerciales en tetrahíbridos de tilapia roja cultivados em Venezuela. Asociación Americana de Soya. Caracas. 33 pp.

Cordeiro, S.A.; da Silva, M.L.; Jacovine, L.A.; Valverde, S.R. e Soares, N.S. 2010. Contribuição do fomento do órgão florestal de Minas Gerais na lucratividade e na redução de riscos para produtores rurais. Rev Árvore, 34: 367-376.

Corrar, J.L. 1993. O modelo econômico da empresa em condições de incerteza aplicação do método de simulação de Monte Carlo. Caderno de Estudos, 8: 1-11.

Furlaneto, F. de P.B.; Ayroza, D.M.M.; Ayroza, R.de e da Silva, L.M. 2006. Custo e rentabilidade da produção de tilápia (Oreochromis spp.) em tanque-rede no médio Paranapanema, Estado de São Paulo, safra 2004/05. Informações Econômicas, 36: 63-69.

Furlaneto, F. de P.B.; Ayroza, D.M.M. de R. e Ayroza, L. M. da Silva. 2010. Análise econômica da produção de tilápia em tanques-rede, ciclo de verão, região do médio Paranapanema, Estado de São. Informações Econômicas, 40: 6-11.

Lima, A.K. dos S; Amancio, A.L.de L.; Casali, R. do R.B.; Santos, L.M. e Rocha, M.M.R.M. 2009. Avaliação técnico-econômica da criação de tilápia nilótica (Oreochromis niloticus) no município de Bananeiras, Estado da Paraíba. T J Fish Aquat Sci, 9: 159-167.

Lira, S.A. e Chaves Neto, A. 2006. Coeficientes de correlação para variáveis ordinais e dicotômicas derivados do coeficiente linear de Pearson. Ciência \& Engenharia, 15: 45-53.

Lyra, G.B.; Ponciano, N.J.; Souza, P.M.; Sousa, E.F. e Lyra, G.B. 2010. Viabilidade econômica e risco do cultivo de mamão em função da lâmina de irrigação e doses de sulfato de amônio. Acta Sci Agron, 32: 547-554.

Maneschy, R.Q.; Santana, A.C. eVeiga, J.B. 2009. Viabilidade econômica de sistemas silvipastoris com Schizolobium parahybavar. amazonicum e Tectona grandis no Pará. Pesqui Florest Bras, 60: 49-56.

Marengoni, N.G. 2006. Produção de tilápia do Nilo Oreochromis niloticus (linhagem chitralada), cultivadas em tanques-rede, sob diferentes densidades de estocagem. Arch Zootec, 55: 127-138.

Martins, J.L.F.; Ferreira, M.L.R.; Pardal, J.M. y Morano, C.A.R. 2012. Comparación de la estimación de la productividad del proceso de soldadura eléctrica por los métodos de simulación de Monte Carlo e Hipercubo Latino. Información tecnológica, 23: 21-32.

Matsumoto, M. and Nishimura, T. 1998. Mersenne Twister: a 623-dimensionally equidistributed uniform pseudorandom number generator. ACM Trans Model Comput Simul, 8: 3-30.

Matsunaga, M.; Bemelmans, P.F. e Toledo, P.E.N. 1976. Metodologia de custo de produção utilizado pelo IEA. Agricultura em São Paulo, 23: 123-139.

Mendonça, T.G.; Lírio, V.S.; Moura, A.D.; Reis, B. dos S. e Silveira, S. de F. 2009. Avaliação da viabilidade econômica da produção de mamão em sistema convencional e de produção integrada de frutas. Rev Econ Nordeste, 40: 699-723.

Murta, R.M.; Veloso, C.M.; Silva, F.F.; Pires, A.J.V. Rocha Neto, A.L. e Costa, L.T.; Santana Júnior, H.A.D. 2013. Viabilidade econômica do uso de fontes lipídicas na dieta de vacas em lactação. Arq Bras Med Vet Zootec, 65: 1454-1462.

Oliveira, R.J. e Pamplona, E. de O. 2012. A volatilidade de projetos industriais para uso em análise de risco de investimentos. Gestão e Produção, 19: 337-345.

Oliveira, R.P. de C.; Silva, P.C.; da Silva, R.F.; Gomes, J.P.; Pádua, D.M.C.; Silveira Filho, P.R.; Machado Júnior, C.C. e Aguiar, M. da S. 2010. Avaliação econômica da produção da tilápia-do-Nilo em tanques com diferentes esquemas de troca de água no sistema raceway. Cienc Anim Bras, 11: 760-763.

Paiva, P.; Mainardes-Pinto, C.S.R.; Verani, J.R. e da Silva, A.L. 2008. Produção da tilápia tailandesa Oreochromis niloticus, estocada em diferentes densidades em tanques-rede de pequeno volume instalados em viveiros de piscicultura povoados ou não com a mesma espécie. Boletim do Instituto de Pesca, 34: 79-88.

Palisade Corporation. 2013. @Risk para Excel. Versão 6.2. Palisade Corporation. Newfield. NY.

Ponciano, N.J., de Souza, P.M.; Mata, H.T.C.; Vieira, J.R. e Morgado, I.F. 2004. Análise de viabilidade econômica e de risco da fruticultura na região norte Fluminense. Rev Econ Socio Rural, 42: 615-635.

Raimundini, S.L.; Bianchi, M. e Zucatto, L.C. 2008. Ponto de equilíbrio e otimização sob a perspectiva da matemática. Enfoque: Reflexão Contábil, 27: 39-55.

Rezaie, K.; Amalnik, M.S.; Gereie, A.; Ostadi, B. and Shakhseniaee, M. 2007. Using extended Monte Carlo simulation method for the improvement of risk management: consideration of relationships between uncertainties. Appl Math Comput, 190: 1492-1501.

Saint-Paul, U. 1986. Potential for aquaculture of South American fresh water fish: a review. Aquaculture, 54: 205-240.

Sanches, E.G.; Tosta, G.A.M. e Souza-Filho, J.J. 2013. Viabilidade econômica da produção de formas juvenis de bijupirá (Rachycentron canadum). Boletim do Instituto de Pesca, 39: 15-26. 
Schwarz, G. 1978. Estimating the dimension of a model. Ann Statistics, 6: 461-464.

Scorvo Filho, J.D.; Mainardes-Pinto, C.S.R.; de Paiva, P.; Verani, J.R. e da Silva, A.L. 2008. Custo operacional de produção da criação de tilápias tailandesas em tanques-rede, de pequeno volume, instalados em viveiros povoados e não povoados. Cust Agronegócio, 4: 98-116.

Siqueira, H.M.; de Souza, P.M. e Ponciano, N.J. 2011. Café convencional versus café orgânico: perspectivas de sustentabilidade socioeconômica dos agricultores familiares do Espírito Santo. Ceres, 58: 155-160.

Silva, J.R.; Rabenschlag, D.R.; Feiden, A.; Boscolo, W.R.; Signor, A.A. e Bueno, G.W. 2012. Produção de pacu em tanques-rede no reservatório de Itaipu, Brasil: retorno econômico. Arch Zootec, 61: 245-254. Simões, D.; Silva, R.B.G. e Silva, M.R. 2012. Composição do substrato sobre o desenvolvimento, qualidade e custo de produção de mudas de Eucalyptus grandis Hill ex Maiden $\times$ Eucalyptus urophylla S.T. Blake. Ciênc Florest, 22: 91-100. 